\title{
Evaluation of dry needling and $0.5 \%$ lidocaine injection therapies in myofascial pain trigger points in masticatory muscles
}

Renato Oliveira Ferreira da Silva', Paulo César Rodrigues Conti ${ }^{2}$, Carlos dos Reis Pereira Araújo ${ }^{3}$, Rafael dos Santos Silva ${ }^{4}$

Objective: The purpose of this study was to compare the effectiveness of trigger points injections using lidocaine $0.5 \%$ and dry needling without any kind of home-based rehabilitation program.

Methods: Sixteen patients with myofascial pain and trigger points in masticatory muscles were randomly assigned to two groups and received only one application session. The pressure pain threshold (PPT) was recorded before and after the injection: Ten minutes, 24 hours later, 7, 15, 21 and 30 days after the treatment. Visual analogue scale (VAS) was used to in all evaluation periods.

Results: There were no difference between groups for PPT, but for all groups the PPT during the time significantly increased when compared the before treatment. VAS showed differences between groups and during the time. The $0.5 \%$ lidocaine had the lowest VAS values when compared to dry needling, but at 30 days there were no differences among them.

Conclusion: Despite the differences in VAS and considering there were no differences in PPT increases, we concluded that, in this study, both groups were able to disrupt the mechanisms of trigger point and relieve the myofascial pain symptoms.

Keywords: Myofascial pain. Trigger point. Needling therapies.

${ }^{1} \mathrm{PhD}$ in Oral Rehabilitation, Bauru Dental School, São Paulo University.

${ }^{2}$ Associate Professor of Department of Prosthodontics and Periodontics, Bauru Dental School, São Paulo University.

${ }^{3} \mathrm{PhD}$ and Professor, Department of Prosthodontics and Periodontics, Bauru Dental School, São Paulo University.

${ }^{4} \mathrm{PhD}$ in Oral Rehabilitation, Bauru Dental School, São Paulo University.
How to cite this article: Silva ROF, Conti PCR, Araújo CRP, Silva RS. Evaluation of dry needling and $0.5 \%$ lidocaine injection therapies in myofascial pain trigger points in masticatory muscles. Dental Press J Orthod. 2012 Mar-Apr;17(2):113-8.

Submitted: November 12, 2007 - Revised and accepted: December 05, 2010

» The authors report no commercial, proprietary, or financial interest in the products or companies described in this article.

Contact address: Renato Oliveira Ferreira da Silva

Rua Dr. Henrique Arouche de Toledo, 1-89 - Bauru / SP - CEP: 17.017-320 - Brazil

E-mail: renatofob@yahoo.com 


\section{INTRODUCTION}

The myogenic disorders have characteristics such as muscle pain (MP) tenderness, limited motion, weakness, among others. These changes may be reactions of changes not originate in the muscles, or process actually originated in the masticatory and cervical muscles. ${ }^{27}$

One of the conditions affecting the muscles and having a high prevalence is the myofascial pain (MFP). This painful process is originated in points of the muscle and due to local and systemic factors, cause muscle pain on both these points and distant structures. ${ }^{6}$ The local muscle tenderness that triggers the process of pain from a distance is called trigger point (TP). The pain it causes in other regions is called referred pain (RP), which can manifest itself in other muscles, teeth, gums, temporomandibular joint (TMJ) and ears. ${ }^{6,9,21}$

The sensitivity of muscle is an important clinical sign, present in most patients with some type of temporomandibular disorder (TMD), reaching a frequency of $88.7 \% .^{21}$ In the literature, this sensitivity is referred to as the pressure pain threshold (PPT) to be, more specifically, the point at which a patient feels that the increasing pressure becomes unpleasant pain sensation. ${ }^{5}$ To assess the muscle sensitivity, the palpation method has been used either manually or with the aid of an algometer. ${ }^{4,24,29}$ Studies that demonstrate the application of algometers for determining the PPT show that these devices are reliable sources and the results are in good to excellent reproducibility, and thus a useful tool to evaluate the initial condition of the patient and also the progress of treatment. ${ }^{13,17}$ The term MP acquired two meanings. One of them is the regional muscular pain associated with muscle tenderness according to Research Diagnostic Criteria (RDC / TMD), and the other on the MP caused by TP which consist of a hyper-focal muscle irritability.

Studies have concluded that the algometer can reliably quantify and measure the TP sensitivity and the active tip of the algometer is important because if not the correct size, can lead to a misinterpretation and a consequent increase in the values read. ${ }^{5,22}$

Another study determined a pressure capable of stimulating pain response in patients with signs and symptoms consistent with TMD, when compared to a control group. Women in both groups were palpated by a single examiner on the following points: Body of masseter, anterior temporal, middle temporal and posterior temporal. We obtained a specificity of $90.8 \%$ for PPT values of $1.5 \mathrm{~kg} / \mathrm{cm}^{2}$ for the masseter, 2.47 for the anterior temporal $\mathrm{kg} / \mathrm{cm}^{2}, 2.75 \mathrm{~kg} / \mathrm{cm}^{2}$ to $2.77 \mathrm{~kg} /$ $\mathrm{cm}^{2}$ and middle temporal for later time. The authors concluded that palpation proved to be a reliable test for detecting muscle tenderness in TMD patients. ${ }^{24}$

Regarding the treatment of trigger points, there are several studies on the effectiveness of each one, dealing only with the needling, injection of anesthetics, injection of corticosteroids and botulinum injection. Reports of success vary and may or may not be complete remission of signs and symptoms. When this happens, some factors may be associated, such as local twitch response (LTR) during the needling and the subsequent increase in PPT., ${ }^{8,12,15,16}$ To sum up, the studies conclude that the injected substances seem to have a role in remission of MP symptoms, always suggesting a more conservative and less costly treatment to patient., ${ }^{2,710,14}$

Due to these factors, this study aims to compare, using algometry and visual analysis scale (VAS), the effectiveness of dry needling in the treatment of patients with MFP, when compared to needle injection of anesthetic solution of lidocaine hydrochloride $0.5 \%$.

\section{MATERIAL AND METHODS}

This study was approved by the Ethics Committee of the Bauru School of Dentistry, University of São Paulo, under protocol No. 174/2004.

The inclusion criteria were intended to exclude factors that could predispose to TMD. ${ }^{20}$ Also excluded were those who presented: History of facial or neck trauma, limitation of movement in the cervical region, active periodontal disease or tooth decay, do not conform to the classification of the RDC / TMD for muscle aches, have made use of anti-inflammatory or analgesic in the last 72 hours, systemic symptoms, physical or mental disability; not agree to participate. After filling out the questionnaire and interview, if the parties did not present any of the above conditions, was carried out physical examination for the diagnosis of TMD.

We collected the personal data of volunteers, chief complaint, history of current condition and medical and dental history. They completed a questionnaire to determine the TMD index, composed of 
10 questions, which allowed the determination of the intensity of the framework of a TMD.

Scores were used: "0" (zero) for no pain, "l" to report mild pain (the patient reports that it hurt a little), " 2 " for moderate pain (patient responds with eyelid reflex) and " 3 "for severe pain (the patient reacts away from the face). At this stage, if the patient was diagnosed with a TP in the masseter or temporal, it was held to fill the form of RDC / TMD.

Some modifications to the RDC / TMD were performed: replaced the palpation of the lateral pterygoid muscle by palpation of the deep portion of masseter, due to clinical and anatomical impossibility of palpation of this muscle ${ }^{25,28,29}$ and digital pressure applied during palpation of the muscles in physical examination was approximately $1.5 \mathrm{~kg}$ for extra-oral muscles instead of 2 pounds $(0.90 \mathrm{~kg})$ and $1 \mathrm{~kg}$ instead of 1 pound $(0.45 \mathrm{~kg})$ to the joint and intraoral muscle. ${ }^{24}$

Algometer was used by the examiner for calibration of the pressure exerted during palpation.

Before distributing the patients in the groups and starting treatment, the VAS and the PPT were measured, so that the patients were allocated in groups presenting the same levels of VAS and similar PPT.

By an algometer, PPT was quantified before treatment (baseline). The values of the time required for maintaining the pressure that cause referred pain, area of manifestation and VAS were recorded.

The groups were named according to the type of treatment and recorded in the patient's general form:

» Group I: Patients who underwent needling and injection of the anesthetic lidocaine hydrochloride $0.5 \%$.

» Group II: Patients who underwent dry needling.

The injections were performed using a $3 \mathrm{ml}$ disposable syringes (BD Plastipak ${ }^{\mathrm{TM}}$ ), Luer Lock ${ }^{\circledR}$ with disposable needles 0,45x13 26 G 1/2 (BD PrecisionGlide ${ }^{\mathrm{rn}}$ ). The amount of anesthetic solution injected was $1 \mathrm{ml} .{ }^{27}$

PPT measurements were made in the following times after the needling: 10 minutes, 24 hours ${ }^{7,15,21}$ and 30 days. A total of seven measurement times were performed. Also, the presence of referred pain and the area of expression were evaluated.

The VAS was assessed before needling (initial) and after, at the following times: 10 minutes, 24 hours, 7, 15, 21 and 30 days.

The patients excluded from the sample or those who still had some symptoms at the completion of the study received the care they need.

All examinations were performed by a single examiner. Before the exams, the examiner underwent training for familiarization with the algometer and standardization of the examination in relation to the rate of force application, pre-set at approximately 0.5 $\mathrm{kg} / \mathrm{cm}^{2} / \mathrm{s}^{18,23,24}$ This step received the supervision of an experienced professional. The examiner was not the same who applied treatment and the patient did not know into which group was inserted.

\section{Statistical analysis}

After collecting the results we applied the two-way ANOVA adopting a significance level lower than 0.05. If there were differences, we applied the Tukey test to detect in which groups there were differences.

\section{RESULTS}

We examined 423 individuals of which 20 female patients were selected. These patients who consent to participate in this study, were presenting myofacial pain according to RDC / TMD, with the presence of $\mathrm{TP}$ in the masseter muscle and referred pain.

After the division of groups, 2 patients expressed wish to not participate further in the study and two others did not appear on the appointed days to return. A total of 16 patients were divided into two equal groups.

\section{PRESSURE PAIN THRESHOLD}

We applied the ANOVA to determine whether there were statistically significant differences between groups. No statistically significant differences were present when comparing the PPT before treatment (initial) between groups ( $p>0.05)$. The PPT had an initial average of $1.05 \mathrm{kgf} / \mathrm{cm}^{2}$.

When the period of data collection and tabulation were finished, we applied the two-way ANOVA. There were no differences between groups, independent of time ( $p>0.05)$.

By observing the interaction between time, regardless of group, there was a statistically significant difference. Then, the Tukey test was applied for multiple comparisons between the periods analyzed (Table 1).

Statistical difference was found between the time before and immediately after treatment. The same difference was presented between times 24 hours and 7, 14 and 30 days, and between 21 days and 
30 days. It means that an increase in the amount of pressure needed to cause pain at the site of palpation was observed (Table 2).

In some patients, the RP process has not been removed, which means the TP structure is still formed. In the group I, there were two patients with RP after 30 days. In group II, there were no patients with RP.

In the VAS evaluation, there was a statistically significant difference between groups and times.

For better understanding, the time difference within each group will be examined initially (Table 3 and 4).

In the comparison between groups within each time, it is seen at the beginning the average of VAS had no differences and significant variation happened between groups over time, but, at the end, there were no differences between the groups I and II.

\section{DISCUSSION}

The mean value obtained for the PPT before treatment in patients with MFP in the study was $1.05 \mathrm{~kg}$ and are partly according to the literature. ${ }^{5}$ There are studies that had higher values, but the areas examined were not the same. However, the authors argue that the PPT is less than in asymptomatic individuals, which agrees with our findings and also with other studies., ${ }^{9,24}$ In our study, the results show no statistically significant differences between groups I and II. These findings are not new if we seek some results in literature. Studies conclude the same results can be satisfactory even changing the injected substance, but these were not performed in the masticatory muscles. ${ }^{8,12,14,26}$

In a systematic review, ${ }^{2}$ it was found that the nature of the injected substance does not matter in the

Table 1 - PPT among groups over the time.

\begin{tabular}{cccccc} 
& PPT_INIT & PPT_IMMED & PPT_24H & PPT_7 & PPT_21 \\
& Mean (s.d.) & Mean (s.d.) & Mean (s.d.) & Mean (s.d.) & Mean (s.d.) \\
\hline Group I & $1.063(0.133)$ & $1.236(0.351)$ & $1.139(0.326)$ & $1.281(0.434)$ & $1.286(0.356)$ \\
Group II & $1.081(0.237)$ & $1.194(0.196)$ & $1.264(0.121)$ & $1.444(0.199)$ & $1.550(0.169)$ \\
\hline
\end{tabular}

Table 2 - PPT over the time regardless group.

\begin{tabular}{cc}
\hline Time & Mean PPT \\
\hline Initial & $1.070278^{a}$ \\
Immediate & $1.197674^{b}$ \\
24 hours & $1.199236^{\mathrm{b}}$ \\
7 days & $1.305799^{\mathrm{bc}}$ \\
14 days & $1.322986^{\mathrm{bc}}$ \\
\hline 21 days & $1.414375^{\mathrm{c}}$ \\
\hline 30 days & $1.573924^{\mathrm{d}}$ \\
\hline
\end{tabular}

Values designated with the same letter are not statistically different.

Table 4 - VAS variance over the time.

\begin{tabular}{cc} 
Time & Mean VAS \\
\hline Initial & $9.4^{\mathrm{a}}$ \\
\hline 24 horas & $8.533334^{\mathrm{a}}$ \\
7 days & $5.233333^{\mathrm{b}}$ \\
\hline 14 days & $5.011111^{\mathrm{b}}$ \\
\hline 21 days & $1.433333^{\mathrm{c}}$ \\
\hline 30 days & $0.122222^{\mathrm{d}}$ \\
\hline
\end{tabular}

Values designated with the same letter are not statistically different.
Table 3 - VAS variance over the time.

\begin{tabular}{|cc|}
\hline Time & Mean VAS \\
\hline Initial & $9.4375^{\mathrm{a}}$ \\
24 hours & $6.8875^{\mathrm{b}}$ \\
\hline 7 days & $4.6875^{\mathrm{c}}$ \\
14 days & $1.1625^{\mathrm{d}}$ \\
\hline 21 days & $0.125^{\mathrm{d}}$ \\
\hline 30 days & $0^{d}$ \\
\hline
\end{tabular}

Values designated with the same letter are not statistically different.

Table 5 - VAS among groups.

\begin{tabular}{ccc} 
& VAS & \\
\hline Time & Group I & Group II \\
\hline Initial & $9.6^{\mathrm{a}}$ & $9.4^{\mathrm{a}}$ \\
24 horas & $6.8875^{\mathrm{c}}$ & $8.533334^{\mathrm{d}}$ \\
7 days & $4.6875^{\mathrm{e}}$ & $5.2333^{\text {ef }}$ \\
\hline 14 days & $1.1625^{\mathrm{g}}$ & $5.011111^{\mathrm{h}}$ \\
\hline 21 days & $0.125^{\mathrm{i}}$ & $1.433333^{\mathrm{i}}$ \\
\hline 30 days & $0^{\mathrm{k}}$ & $0.122222^{\mathrm{k}}$ \\
\hline
\end{tabular}

Values designated with the same letter are not statistically different. 
final result. Dry needling is as effective as needling with the inoculation of some kind of substance. They found no convincing scientific evidence that needling has an effect beyond placebo in myofascial pain. ${ }^{2}$ This study also comments on the limitations of the study as not specify whether patients had TP. Another aspect is in relation to diagnostic criteria, which would be the combination of muscle tenderness in a palpable band of a skeletal muscle and the onset of pain, stating that the effect of the emergence of LTR appears to be more important than the type of substance injected, but this type of analysis was only considered in a single study. The authors ${ }^{2}$ conclude by saying that although one can not scientifically say that there is some advantage in using the needle, regardless of whether or not injecting some kind of substance, it is recommended to its implementation, since it is the most safe and comfortable to the patient. ${ }^{2}$

Although there are no differences between the groups when examined separately and over time, while comparing the values of PPT it was noticed an increase in the amount of pressure necessary to have a painful site process. This painful process in most of the time was not accompanied by the presence of RP anymore. Other studies support our findings, in that there be an increased PPT and also in some cases, no change thereof. Relief of TP symptoms occurs regardless of whether or not applying lidocaine. They noted that it was more important to obtain the LTR, as in cases that do not got this answer, no remission of symptoms was perceived. Moreover, in cases that had some type of RP process, may have been stimulation of latent TP, which can occur when we provoke the trauma generated by the needle. ${ }^{8,11,16}$ This factor may explain the fact that at the end of the study some patients had the RP process even if the pressure was higher than those recorded in previous periods. Another fact is that only one injection was performed, which may not have been enough to neutralize all satellites TP and also no patient counseling was perform, such as stretching, hot packs or other methods that are cited in literature. Regarding the increase in the PPT immediately observed other studies support it, but often the patient stills reporting the referred pain, which is equal in the experiment.11,15 This phenomenon can occur because there may be the existence of satellites TP around the injected TP. These points would then not eliminated by the process and due to mechanical trauma, these satellites TP can be activated, which leads to maintenance of the RP and often not change PPT.,10,11,16

The initial PPT averaged was $1.05 \mathrm{~kg}$, and reached the final values of $1.57 \mathrm{~kg}$. This final value is close to normal in healthy individuals, about 1.5 $\mathrm{kg}$ for masseter muscle. ${ }^{24}$ Other studies also say that healthy muscles have higher PPT than those muscles affected by MP. ${ }^{16,18,22}$

When assessing the results of the VAS of pain, the findings were somewhat different. Regarding the decreased level of pain scored by patients, all groups had a statistically significant decrease over time, and all groups reached values equal to zero, if not close it, or came from the vicinity of the "worst pain imaginable" to close the "no pain". This decrease is consistent with the increase of the PPT, because if there was a remission of symptoms, there was a real effectiveness of treatment and consequent decreased sensation of pain experienced by patients, and this was shown by other studies. ${ }^{7,11,13}$ A decrease between 28 and $22 \mathrm{~mm}$ on the VAS reading was enough to show the effectiveness of the therapy. These findings may be extrapolated to a population with MFP in general. The decreasing of 20-30 mm in VAS relative to the baseline may be clinically relevant to successful treatment. ${ }^{3}$ This finding is confirmed on the results. ${ }^{1,30}$

\section{CONCLUSION}

Based on the results that were presented, it is possible to conclude that there are no differences between the applied treatments for the relief of MFP and that all groups decreased the patient's sensation of pain measured by VAS and increased the PPT, even in a short period evaluation. The presence of TP related symptoms in some patients indicates that the treatment may require more than a single approach of injections or interaction with other specialties. It would be interesting in future studies to carry out placebo groups for the verification of its effect on patient response. 


\section{REFERENCES}

1. Conti PC, de Azevedo LR, de Souza NV, Ferreira FV. Pain measurement in TMD patients: evaluation of precision and sensitivity of different scales. J Oral Rehabil. 2001;28(6):534-9.

2. Cummings TM, White AR. Needling therapies in the management of myofascial trigger point pain: a systematic review. Arch Phys Med Rehabil. 2001;82:986-92.

3. De Laat A, Stappaerts K, Papy S. Counselin and physical therapy as treatment for myofascial pain of the masticatory system. J Orofc Pain. 2003;17(1):42-9.

4. Dworking SF, LeResche L. Research diagnostic criteria for temporomandibular disorders: review, criteria, examinations and specifications, critique. J Craniomandib Disord. 1992;6(4):301-55.

5. Fischer AA. Pressure algometry over normal muscles. Standard values, validity and reproducibility of pressure threshold. Pain. 1987;30(1):115-26.

6. Fricton JR. Myofascial pain syndrome of the head and neck: a review of clinical characteristics of 164 patients. Oral Surg Oral Med Oral Pathol. 1985;60(6):615-23.

7. Graboski CL, Gray DS, Burnhan RS. Botulinum toxin A versus bupivacaine trigger point injections for the treatment of myofascial pain syndrome: a randomized double blind crossover study. Pain. 2005;118(1-2):170-5. Epub 2005 Oct 3.

8. Hong CZ. Lidocaine injection versus dry needling to myofascial trigger point. The importance of the local twitch response. Am J Phys Med Rehabil. 1994;73(4):256-63.

9. Hong CZ. Pressure threshold for referred pain by compression on the trigger point and adjacent areas. J Musculosk Pain. 1996;4(3):61-79.

10. Hong CZ. Treatment of myofascial pain syndrome. Curr Pain Headache Rep. 2006;10(5):345-9.

11. Hsieh YL, Kao MJ, Kuan TS, Chen SM, Chen JT, Hong CZ. Dry needling to a key miofascial trigger point may reduce the irritability of satellite miofascial trigger points. Am J Phys Med Rehabil. 2007;86(5):397-403.

12. I wama $\mathrm{H}$, Akama $\mathrm{Y}$. The superiority of water-diluted $0.25 \%$ to neat $1 \%$ lidocaine for trigger-point injections in myofascial pain syndrome: a prospective, randomized, double-blinded trial. Anesth Analg. 2000;91(2):408-9.

13. Jaeger B, Reeves JL. Quantification of changes in myofascial trigger point sensitivity with the pressure algometer following passive stretch. Pain. 1986;27(2):203-10.

14. Kamanli A, Kaya A, Ardicoglu O, Ozgocmen S, Zengin FO, Bayik Y. Comparison of lidocaine injection, botulinum toxin injection, and dry needling to trigger points in myofascial pain syndrome. Rheumatol Int. 2005;25(8):604-11. Epub 2004 Sep 15

15. Lewit K. The needle effect in the relief of myofascial pain. Pain. 1979;6(1):83-90.
16. McMillan AS, Blasberg B. Pain-Pressure threshold in painful jaw muscles following trigger points injection. J Orofac Pain. 1994;8(4):384-90.

17. Mense S, Simons DG. Myofascial pain caused by trigger points. In: Mense S, Simons DG. Muscle pain: understanding its nature, diagnosis, and treatment. Philadelphia: Lippincott Williams \& Wilkins; 2001. p. 205-88.

18. Ohrbach R, Gale EN. Pressure pain thresholds, clinical assessment, and differential diagnosis: reliability and validity in patients with myogenic pain. Pain. 1989;39(2):157-69.

19. Okeson JP. Nonodontogenic toothache. Dent Clin North Am. 1997;41(2):367-83.

20. Pullinger AG, Seligman DA, Dornbein JA. A multiple logistic regression analysis of the risk and relative odds of temporomandibular disorders as a function of common occlusal features. J Dent Res. 1993;72(6):968-79.

21. Reeh ES, el-Deeb ME. Referred pain of muscular origin resembling endodontic involvement. Oral Surg Oral Med Oral Pathol. 1991;71(2):223-7.

22. Reeves JL, Jaeger B, Graff-Radford SB. Reliability of the pressure algometer as a measure of myofascial trigger point sensitivity. Pain. 1986;24(3):313-21.

23. Reid KI, Gracely RH, Dubner RA. The influence of time, facial side, and location on pain-pressure thresholds in chronic myogenous temporomandibular disorder. J Orofac Pain. 1994:8(3):258-65.

24. Silva RS, Conti PC, Lauris JR, Silva RO, Pegoraro LF. Pressure pain threshold in the detection of masticatory myofascial pain: an algometer-based study. J Orofac Pain. 2005;19(4):318-24

25. Stratmann U, Mokrys K, Meyer U. Clinical anatomy and palpability of the inferior lateral pterygoid muscle. J Prosthet Dent. 2000;83(5):548-54.

26. Travell JG. Temporomandibular joint pain referred from muscles of the head and neck. J Prosthet Dent. 1960:10(4):745-63.

27. Travell JG, Simons DG. Background and principles. In: Travell JG, Simons DG. Myofascial pain and dysfunction. The trigger point manual. Baltimore: Williams \& Wilkins; 1983. p. 5-44.

28. Türp J, Minagi S. Palpation of the lateral pterygoid region in TMD - where is the evidence? J Dent. 2001:29(7):475-83.

29. Visscher CM, Lobbezoo F, Naeije M. Comparison of algometry and palpation in the recognition of temporomandibular disorder pain complaints. J Orofac Pain. 2004;18(3):214-9.

30. Williamson A, Hoggart B. Pain: a review of three commonly used pain rating scales. J Clin Nurs. 2005:14(7):798-804 